\title{
Preparation of nobiletin in self-microemulsifying systems and its intestinal permeability in rats
}

\author{
Jing Yao, Yun Lu, Jian Ping Zhou \\ Department of pharmaceutics, China Pharmaceutical University, Nanjing, People's Republic of China
}

Received, December 11, 2007; Revised, May 29, 2008; Accepted, June 9, 2008; Published, June 18, 2008.

\begin{abstract}
PURPOSE. The objective of this study was to prepare nobiletin self-microemulsifying drug delivery systems (SMEDDS) and investigate its intestinal transport behavior using the single-pass intestinal perfusion (SPIP) method in rat. METHODS. The characterizations of nobiletin SMEDDS were investigated. SPIP was performed in each isolated region of the small intestine (i.e. duodenum, jejunum, ileum and colon) over three concentrations of nobiletin $(15,30$ and $60 \mu \mathrm{g} / \mathrm{mL})$ at a flow rate of $0.2 \mathrm{ml} / \mathrm{min}$. The concentrations of the samples were determined by HPLC and the effective permeability coefficients $\left(P_{\text {eff }}\right)$ in rats were calculated. Considering the high correlation of rat $P_{\text {eff }}$ values with those of human, the human intestinal permeability was predicted using the Lawrence compartment absorption and transit model. The intestinal permeability of nobiletin in SMEDDS, sub-microemulsions and micelles was compared. RESULTS. The particle size and zeta potential of nobiletin SMEDDS were $(28.6 \pm 0.3) \mathrm{nm}$ and $(-22.6 \pm 3.5) \mathrm{mV}$, respectively. The $P_{\text {eff }}$ in jejunum at $15 \mu \mathrm{g} / \mathrm{mL}$ was significantly higher than that at 60 $\mu \mathrm{g} / \mathrm{mL}(\mathrm{p}<0.01)$. The $P_{\text {eff }}$ in colon was higher at the same concentration comparing to the other intestinal segments. Moreover, there was no statistical difference in $P_{\text {eff }}$ at each same concentration in jejunum, duodenum and ileum. The estimated human absorption of nobiletin for the SMEDDS dilutions was higher than that for sub-microemulsions $(p<0.01)$ and similar to that of the micelles $(p>0.05)$. CONCLUSIONS. Bases on the above results, the SMEDDS could enhance the intestinal permeability of the nobiletin, and may be presented as potential candidates for improving the oral absorption of the noblietin.
\end{abstract}

Corresponding Author: Dr. Jian Pin Zhou, E-mail Address: zhoujpcpu@126.com

\section{INTRODUCTION}

In recent years, much attention has been focused on oral dosage forms using a self-microemulsifying drug delivery system (SMEDDS) for the purpose of improving the solubility and absorption of poorly water-soluble drugs. SMEDDS consists of a mixture of drugs, oils, surfactants and/or other additives. Gentle mixing of these ingredients in aqueous media generates microemulsions with a droplet size in a range of 10-100 $\mathrm{nm}$. SMEDDS has been shown to improve absorption of drugs by rapid self-microemulsification in the stomach, with the microemulsion droplets subsequently dispersing in the gastrointestinal tract to reach sites of absorption [1]. The resultant small droplet size from SMEDDS provides a large interfacial surface area for drug release and absorption, and the specific components of SMEDDS promote the intestinal lymphatic transport of drugs [2]. Oral absorption of several drugs has been enhanced by SMEDDS [2-5]. Sandimmu Neoral ${ }^{\circledR} \quad$ (CsA-NEO), a self-microemulsifying capsule of cyclosporine A, had been approved by FDA.

Nobiletin (5, 6, 7, 8, 3', 4'-hexamethoxy flavone), Figure 1, a citrus polymethoxy flavonoid, is a candidate of the anti-inflammatory drug [6,7]. A number of studies have shown it to be effective in the treatment and precaution of stomach cancer [8-10]. It may also be effective as a sunscreen reagent for protection against photoinflammation and photoaging [11]. It is known that intestinal permeability is necessary for oral administration [12]. Because the nobiletin is a poor water-soluble drug, it has a very low absolute bioavailability (about 4\%).

In this study, we developed an optimized formulation using SMEDDS to improve the solubility and oral absorption of nobiletin. 


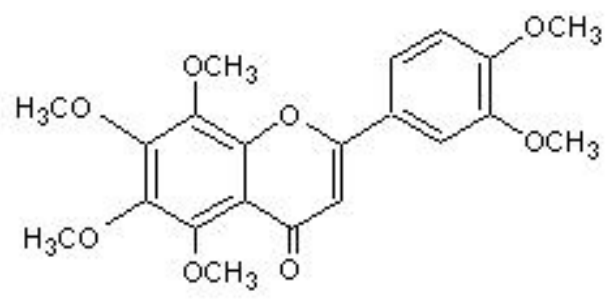

Figure 1. Structure of nobiletin $\left(5,6,7,8,3^{\prime}, 4^{\prime}\right.$ - -hexamethoxyflavone)

The 1:1 mixture of polyoxyethylene 35 castor oil and polysorbate 80 was used as the surfactant, polyethylene glycol 400 (PEG 400) as the co-surfactant and the 3:1 mixture of medium-chain triglyceride/tea oil as the oil phase in the formulations.

The intestinal permeability of the nobiletin was determined using the single pass intestinal perfusion (SPIP) in rats. The SPIP method provides the unique advantages of experimental control (e.g. compound concentration and intestinal perfusion rate), ability to study regional differences and an intact intestinal blood supply and a functional intestinal barrier [12-14].

\section{MATERIALS AND METHODS}

\section{Materials}

Nobiletin was obtained from Chinabest Drugs Research Ltd. (Nanjing, China). Polyoxyethylene 35 castor oil USP24/NF19 (PEG-35 castor oil, Cremophor EL 35) was purchased from BASF (Ludwigshafen, Germany). Medium-chain triglyceride (Caprylic/capric triglyceride, Cradamol GTCC) was a kind gift from CRODA (Singapore). Polysorbate 80 (Tween 80) was purchased from Jiangsu Chenpai Pharmaceutical Co., Ltd. (Haimen, China). Polyethylene glycol 400 (PEG 400) was purchased from Beijing Haidian Huiyou Refined Chemical Plant (Beijing, China).Other chemicals were of HPLC or analytic grade.

\section{Animals}

Sprague-Dawley male rats $(180-220 \mathrm{~g})$ from Experimental Animal Center of China Pharmaceutical University were used in this paper. The protocol was approved by Local Animal Ethics Committee.

\section{Preparation of nobiletin self-microemulsifying system}

A mixture of the surfactants, co-surfactant and oils were prepared at a fixed ratio of 7:2:1 (g/g). Nobiletin was then dissolved in the resulting mixture by stirring magnetically at $40^{\circ} \mathrm{C}$ water bath Before the SPIP experiment, sufficient Krebs-Ringer's buffer solution [15] was added to adjust the formulations to a designed concentration.

The intestinal permeability experiments of the SMEDDS solutions with different concentrations of the nobiletin were investigated and compared to both the micelle solution of equivalent surfactant and co-surfactant concentration and the sub-microemulsion of the 0.78:0.22:1 surfactants/ co-surfactant /oils.

\section{Particle size and zeta potential measurement}

The formulation $(0.5 \mathrm{~mL})$ of SMEDDS was diluted with double-distilled water to $10 \mathrm{~mL}$. Photo correlation spectrometer (Zetasizer 3000HS, Malvern Instruments Corp., U.K.) was employed to measure particle sizes and zeta potential of the SMEDDS dilutions, sub-microemulsions and micelles.

\section{Binding and stability studies}

Preliminary studies are necessary before commencing the SPIP studies to ensure that the loss of drug from perfusion is due to absorption and not by other losses (e.g. the binding of the intestinal wall or degradation).

Rat intestinal segments of approximately $10 \mathrm{~cm}$ size were clipped. The intestinal sac were turned over and put into $50 \mathrm{~mL}$ Krebs-Ringer's buffer solution, in which nobiletin $(30 \mu \mathrm{g} / \mathrm{mL})$ was incubated at $37^{\circ} \mathrm{C}$ for $3 \mathrm{~h}$. Samples $(50 \mu \mathrm{L})$ were 
removed at $3 \mathrm{~h}$, and were diluted with absolute methanol, and assayed by HPLC.

Nobiletin was also incubated in $10 \mathrm{~mL}$ perfused Krebs-Ringer's buffer for the intestinal segment at $37^{\circ} \mathrm{C}$ for $3 \mathrm{~h}$. Samples $(50 \mu \mathrm{L})$ were removed at 60 , 120 and $180 \mathrm{~min}$ and diluted with an appropriate volume of absolute methanol, and assayed by HPLC.

\section{Single-pass perfusion of the rat intestinal segments}

SPIP studies were performed according to previously described methods [12,16]. After an overnight fast, male SD rats weighing approximately 180-220 g (Central Animal Laboratory of China Pharmaceutical University, China) were anesthetized with an i.p. injection of $20 \%(\mathrm{w} / \mathrm{v})$ urethane solution (urethane: $1 \mathrm{~g} / \mathrm{kg}$ ). To maintain normal body temperature, rats were placed on a warming pad under a surgical lamp. The abdomen was opened with a midline incision, an interest intestinal segment of approximately $10 \mathrm{~cm}$ was chosen and cannulated with plastic tubing. Initially, the intestinal segment was rinsed with isotonic saline $\left(37^{\circ} \mathrm{C}\right)$ until the outlet solution was clear. Using an infusion pump (FB-1, Kingdom Biochemical Instrument Co., Ltd., Shanghai, China), the intestinal segments were perfused at a flow rate of $0.2 \mathrm{ml} / \mathrm{min}$ with the bland Krebs-Ringer's buffer solution. The preparation time took approximately $30 \mathrm{~min}$. Then the pump was connected to the reservoir containing the nobiletin-loaded SMEDDS dilutions with the marker (Phenol Red).

Setting $t=0$ when the buffer solution has been completely pushed out. Each perfusion experiment lasted for $180 \mathrm{~min}$. and the perfusate was quantitatively collected in the following intervals: $0-60, \quad 60-75, \quad 75-90, \quad 90-105, \quad 105-120$, $120-135,135-150,150-165$ and $165-180 \mathrm{~min}$. The steady state reached in $60 \mathrm{~min}$.

Samples were stored in a refrigerator at $4^{\circ} \mathrm{C}$ until analysis by HPLC.

\section{HPLC analysis of samples}

All samples were diluted to $5 \mathrm{ml}$ with absolute methanol and measured by HPLC. Aliquots of 20 $\mu \mathrm{L}$ were injected onto the HPLC system, equipped with a Shim-pack CLC-ODS column $(5 \mu \mathrm{m}, 150 * 4$ mm, Kyoto, Japan). The HPLC system (LC-2010C,
SHIMADZU Corp., Kyoto, Japan) consisted of an auto-sampler and an ultraviolet detector. The detection wavelength was set at $332 \mathrm{~nm}$. The mobile phase was a methanol-water $(80: 20, \mathrm{v} / \mathrm{v})$ mixture with the flow rate of $1.0 \mathrm{ml} / \mathrm{min}$.

\section{Data Analysis}

Water absorption or secretion (flux) was measured by methods: gravimetric, phenol red, and ${ }^{14} \mathrm{C}$ PEG-3500 [17]. The net water flux (NWF) per $\mathrm{cm}$ of each segment was calculated using Equation (1) [18]:

$$
\begin{gathered}
\mathrm{NWF}\left(\mu \mathrm{l} \cdot \mathrm{min}^{-1} \cdot \mathrm{cm}^{-1}\right)=\left(1-\mathrm{CPR}_{\text {out }} / \mathrm{CPR}_{\text {in }}\right)^{*} \mathrm{Q}_{\text {in }} \\
/ \mathrm{L}^{*} 1000
\end{gathered}
$$

Where $\mathrm{CPR}_{\text {in }}$ and $\mathrm{CPR}_{\text {out }}$ were the inlet and outlet concentration of phenol red, respectively. $Q_{\text {in }}$ was the flow rate of the perfusion solution entering the intestinal segment, $\mathrm{L}$ was the length of the intestinal segment $(\mathrm{cm})$.

Under most conditions, NWF is negative value, which indicates the intestinal lumen would absorb the water. Only when the high osmotic solution is administered, the intestinal lumen secretes the water and UWF is positive value $[17,19,20]$.

The effective permeability, $P_{\text {eff }}$ was calculated based on the inlet and outlet concentration of nobiletin $\left(\mathrm{C}_{\text {in }}\right.$ and $\mathrm{C}_{\text {out }}$, respectively). Steady state was considered to have been achieved when the concentration of phenol red and nobiletin were constant. The permeability values are calculated only from experiments where steady state was achieved.

The permeability was calculated using Equation (2) $[12,17]$ :

$$
P_{\text {eff }}=\frac{Q \ln \left(C_{\text {in }} / C_{\text {out }}\right)^{\prime}}{2 \pi r L}
$$

Where $\mathrm{Q}$ is the flow rate in $\mathrm{ml} / \mathrm{min}, \mathrm{r}$ the radius of the intestine $(0.18 \mathrm{~cm}$ in rat and $1.75 \mathrm{~cm}$ in man), $\mathrm{L}$ is the length of the perfused intestinal segment $(\mathrm{cm})$, and $\left(\mathrm{C}_{\mathrm{in}} / \mathrm{C}_{\text {out }}\right)$ is the concentration ratio corrected for fluid flux.

The statistical significance of the differences among group means were assessed using the one-way unweighted means analysis of variance (ANOVA) with the least significant difference (LSD) test and a value of $\mathrm{p}<0.05$ was considered statistically significance. 


\section{RESULTS}

\section{SMEDDS formulations}

SMEDDS formulations should be clear, monophasic liquid at ambient temperature, and should have suitable solvent properties to allow presentation of drug in solution [3]. Polyoxyethylene 35 castor oil and polysorbate 80 were used as the surfactants because of the higher solubility of nobiletin in polyoxyethylene 35 castor oil and polysorbate 80 observed. Caprylic/capric triglyceride (GTCC), medium-chain triglyceride, was selected as the oil phase for formulation development because it provided higher solubility than other oils and might influence the tight junctions of the epithelial cells [21]. Moreover, to obtain the more stable microemulsions with smaller particle size after self-microemulsification, the tea oil was selected as the other oil. The tea oil, the mixture consisting long chain fatty acids, stimulates the lipoprotein synthesis and subsequent lymphatic absorption [22]. According to the results of the pseudo-ternary phase diagram in the previous study, the 3:1 mixture of medium-chain triglyceride/tea oil as the oil phase was selected. PEG400 was introduced to the system as the co-surfactant for its higher solubilization ability of nobiletin and its low toxicity. The optimal formulation of SMEDDS was selected regarding solubilization ability, self-microemulsifying ability (including the size and stability of microemulsions after self-microemulsification) and reduced use of emulsifiers. The nobiletin SMEDDS were stable for at lest 6 months at ambient temperature.

\section{Particle sizes and zeta potential of three preparations}

The particle sizes and zeta potential of the SMEDDS dilutions, sub-microemulsions and micelles are shown in Table 1. The sub-microemulsions had a larger particle size and wider size distribution compared to the SMEDDS dilutions and micelles. According to the zeta potential values, three preparations had negative charge.

\section{Validation of HPLC assay}

The regression equation for the concentration of nobiletin $(\mathrm{ng} / \mathrm{mL})$ vs response in the perfusion fluid ranging from $80 \mathrm{ng} / \mathrm{mL}$ to $800 \mathrm{ng} / \mathrm{mL}$ was $\mathrm{A}=95.88 \mathrm{C}+820.3(\mathrm{r}=0.9999)$. The mean recovery of nobiletin was $101.3 \pm 1.36 \%$. The within-day and between-day RSD's were less than 3\%.

\section{Binding and stability studies}

Nobiletin did not exhibit markedly binding to the intestinal wall. The remaining amount of nobiletin after incubated in the Krebs-Ringer's buffer with the intestinal sac for $3 \mathrm{~h}$ was $99.43 \pm 3.34 \%$, which was not significantly decreased.

The stability of nobiletin was based on the decrease drug concentration as quantitated by HPLC. After $3 \mathrm{~h}$ incubation, at least $98.2 \%$ nobiletin remained in the perfused Krebs-Ringer's buffer for each intestinal segment. Neither of the potential metabolites was observed in the chromatograms.

\section{Water absorption or secretion of intestinal lumens}

The NWF of each segment is listed in Table 2. The NWF for all intestinal segments were negative value, which suggested that the water was mainly absorbed in the intestine tract. The NWF for the duodenum was significantly higher than that for the jejunum, ileum and colon $(\mathrm{P}<0.05)$, but variance in NWF for the jejunum, ileum and colon were not significant $(\mathrm{P}>0.05)$.

Table 1. Characterizations of the SMEDDS, sub-microemulsions and micelles $(n=3)$.

\begin{tabular}{lccc}
\hline Parameters & SMEDDS & Sub-microemulsions & Micelles \\
\hline Particle size $(\mathrm{nm})$ & $28.6 \pm 0.3$ & $184.9 \pm 3.5$ & $10.1 \pm 1.5$ \\
Polydispersity index & $0.068 \pm 0.026$ & $0.547 \pm 0.010$ & $0.086 \pm 0.021$ \\
Zeta potential $(\mathrm{mV})$ & $-22.6 \pm 3.5$ & $-28.0 \pm 6.4$ & $-20.7 \pm 4.2$ \\
\hline
\end{tabular}


Table 2. The net water flux (NWF) in each intestinal segment $(n=4)$

\begin{tabular}{lcccc}
\hline Time (min) & Duodenum & Jejunum & Ileum & Colon \\
\hline 75 & $-0.584 \pm 0.439$ & $-0.144 \pm 0.082$ & $-0.009 \pm 0.228$ & $-0.146 \pm 0.127$ \\
90 & $-0.555 \pm 0.564$ & $-0.117 \pm 0.121$ & $-0.238 \pm 0.553$ & $-0.197 \pm 0.103$ \\
105 & $-0.714 \pm 0.241$ & $-0.341 \pm 0.199$ & $-0.129 \pm 0.372$ & $-0.237 \pm 0.037$ \\
120 & $-0.795 \pm 0.185$ & $-0.209 \pm 0.454$ & $-0.237 \pm 0.262$ & $-0.426 \pm 0.176$ \\
135 & $-0.666 \pm 0.414$ & $-0.206 \pm 0.119$ & $-0.340 \pm 0.444$ & $-0.298 \pm 0.110$ \\
\hline
\end{tabular}

\section{SPIP studies}

The intestinal permeability of nobiletin was studied as a function of concentration in each segment of the intestine. The upper boundary $(60 \mu \mathrm{g} / \mathrm{mL})$ of the concentration range was chosen because of the limited aqueous solubility of nobiletin.

Steady state is confirmed by plotting the concentration of nobiletin and phenol red versus time in Figure 2. Steady state, which was assessed by the constant concentrations of both phenol red and nobiletin, was reached about $60 \mathrm{~min}$ after the beginning of the perfusion. The permeability values are calculated only from experiments where steady state was achieved.

The permeability values of SMEDDS dilutions for each intestinal segment and concentrations are listed in Table 3. There appears to be a trend of decreasing permeability with increasing concentration. $P_{\text {eff }}$ in the jejunum at $15 \mu \mathrm{g} / \mathrm{mL}$
$(0.445 \pm 0.026 \mathrm{~cm} / \mathrm{s})$ was significantly higher than $P_{\text {eff }}$ at $60 \mu \mathrm{g} / \mathrm{mL} \quad(0.377 \pm 0.057 \mathrm{~cm} / \mathrm{s}, \quad \mathrm{p}<0.01)$. Moreover, there was no statistical difference in $P_{\text {eff }}$ at three investigated concentrations in other segments (including duodenum, ileum and colon).

Comparisons across each segment revealed that the permeability at each concentration in colon was higher than all other segments $(\mathrm{p}<0.01)$. Moreover, the permeability values in duodenum, jejunum and ileum were not statistically different ( $p>0.05)$.

Figure 3 shows the permeability values of nobiletin in the formulation of SMEDDS dilutions, micelle and sub-microemlsion for each segment at $30 \mu \mathrm{g} / \mathrm{mL}$. $P_{\text {eff }}$ of SMEDDS dilutions and micelle in each segment was significantly higher than that of sub-micreomulsions $\quad(\mathrm{p}<0.01)$. Except the duodenum $(\mathrm{p}<0.05), P_{\text {eff }}$ of SMEDDS dilutions and micelle in the jejunum, ileum and colon did not all reach statistical difference $(\mathrm{p}>0.05)$

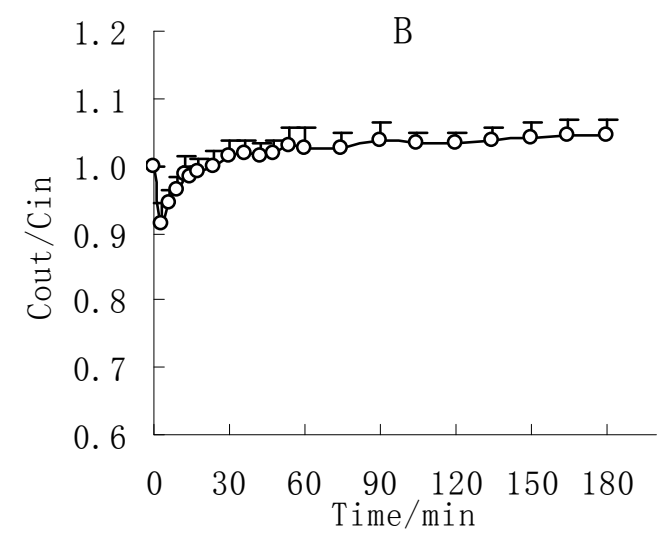

Figure 2. Representative plot of the concentration ratio of the outlet and inlet concentrations $\left(C_{\text {out }} / C_{\text {in }}\right) v s$. time. Phenol red in perfusion solution (B); Drug apparent absorption fraction curve (A). 
Table 3. Comparison of effective permeation coefficients $\left(P_{\text {eff }} \times 10^{-4}\right)$ of different intestinal segments with three concentrations. Results are given as mean \pm s.d. $(n=4)$.

\begin{tabular}{lcccc}
\hline $\begin{array}{c}\text { Conc. of nobiletin } \\
(\mu \mathrm{g} / \mathrm{mL})\end{array}$ & Duodenum & Jejunum & eff & $(\mathrm{cm} / \mathrm{s})$ \\
& $0.435 \pm 0.049$ & $0.445 \pm 0.026$ & $0.475 \pm 0.052$ & $0.625 \pm 0.096$ \\
15 & $0.402 \pm 0.045$ & $0.414 \pm 0.042$ & $0.456 \pm 0.067$ & $0.598 \pm 0.085$ \\
60 & $0.401 \pm 0.068$ & $0.377 \pm 0.057$ & $0.437 \pm 0.116$ & $0.532 \pm 0.068$ \\
\hline
\end{tabular}

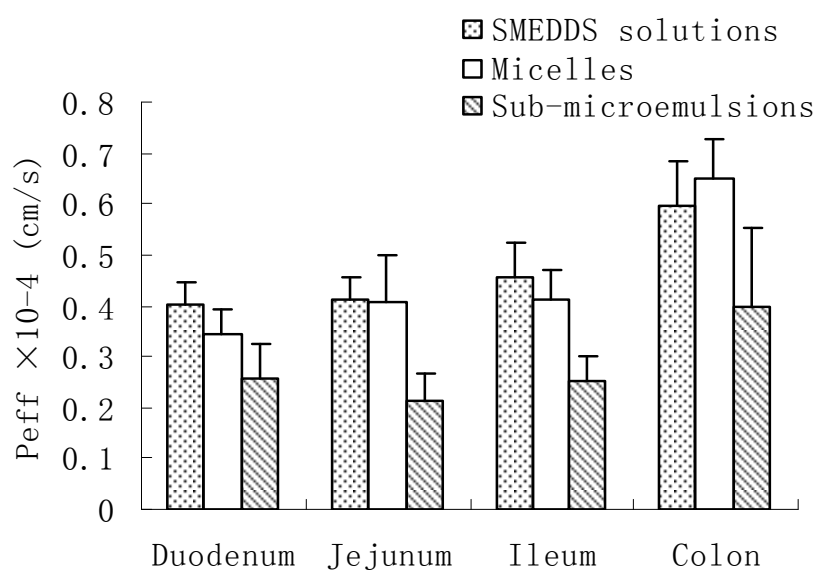

Figure 3. Comparison of effective permeation coefficient $\left(P_{\text {eff }}\right)$ of the SMEDDS solutions, sub-microemulsions and micelles in different intestinal segments $(\mathrm{n}=4)$. The concentration of the nobiletin in the perfusion solutions was $30 \mu \mathrm{g} / \mathrm{mL}$.

\section{DISCUSSIONS}

According to $P_{\text {eff }}$ values in each intestinal segment (shown in Table 3 ), the $P_{\text {eff }}$ at each concentration in the colon was significantly higher than those in other segments, which is consistent with previous studies [23-25]. As a multi-drug resistance-reversing agent [26], the intestinal absorption of nobiletin was limited due to the presence of the P-gp throughout the intestinal tract. There are various reports showing the regional difference in the expression and activity of P-gp throughout the gastric intestinal tract [27]. The order of the expression and activity of P-gp is ileum $>$ duodenum and jejunum $>$ proximal and distal colon [27]. The lower expression activity of P-gp conduced to be increasing the permeability of nobiletin in colon as compared to other sections (Table 3). Moreover, the SMEDDS dilutions, sub-microemulsion and micelle had native charge. It has been shown that there is a selectivity of permeation depending of the gut sections. The small intestine has more cationic selectivity, whereas, the colon has more anionic selectivity [29]. Another possible mechanism for higher $P_{\text {eff }}$ values in colon was that the higher crypt surface area in the colonic mucosa compared with the small intestine could account for its high paracellular permeability [29].

Linear correlation of intestinal permeability and the fraction absorbed between human and rats has been reported for various drugs [30, 31]. The extent of drug absorption in human can be predicted from rat intestinal permeability experiments. Human intestinal permeability values may be estimated using the Lawrence compartmental absorption and transit (CAT) model $[18,32]$ :

$$
\begin{aligned}
& \mathrm{F}_{\mathrm{a}}=1-\left[1+P_{\text {eff, } \operatorname{man}} \times 0.54\right]^{-7} \\
& P_{\text {eff, } \operatorname{man}}=3.6 \times P_{\text {eff, rat }}+0.03 \times 10^{-4}
\end{aligned}
$$

According to this equation (3) and (4), the estimated $\mathrm{F}_{\mathrm{a}}$ for the nobiletin in the SMEDDS dilutions, sub-microemulsions and micelles were $83.5 \pm 2.8 \%, 63.0 \pm 7.1 \%$ and $82.5 \pm 4.9 \%$, respectively. It suggested that the estimated oral absorption in 
human for SMEDDS dilutions would be higher than that for sub-microemulsions $(\mathrm{p}<0.01)$, and similar with that for the micelles $(p>0.05)$. The lower oral absorption for the sub-microemulsions may be related to its larger particle size with wider size distribution (Polydispersity index in table 1: $0.547 \pm 0.010$ ), as suggested in the previous studies, where a better absorption profile was observed after oral administration of the microemulsions containing cyclosporine [33, 34]. SMEDDS are known to improve the oral absorption of lipophilic drugs. The main mechanism reported include increasing membrane fluidity, opening tight junction, inhibiting P-gp and/or CYP450 by surfactants and stimulating lipoprotein/chylomicron production by lipid $[2,35]$. Similar oral absorption of the SMEDDS and micelles showed that the surfactant-induced membrane fluidity and inhibition of P-gp might play an important role in the permeability change and the increase of drug

\section{REFERENCES}

[1]. Shah NH, Carvajal MT, Patel CI, Infeld MH, and Malick AW. Self-emulsifying drug delivery systems (SEDDS) with polyglycolyzed glycerides for improving in vitro dissolution and oral absorption of lipophilic drugs. Int J Pharm, 106: 15-23,1994.

[2]. Wu W, Wang Y, and Que L. Enhanced bioavailability of silymarin by selfmicroemulsifying drug delivery system. Eur $\mathbf{J}$ Pharm Biopharm, 63: 288-294,2006.

[3]. Kommuru TR, Gurley B, Khan MA, and Reddy IK. Self-emulsifying drug delivery systems (SEDDS) of coenzyme Q10: formulation development and bioavailability assessment. Int $\mathbf{J}$ Pharm, 212 : 233-246,2001.

[4]. Itoh K, Matsui S, Tozuka Y, Oguchi T, and Yamamoto K. Improvement of physicochemical properties of N-4472 Part II: characterization of $\mathrm{N}-4472$ microemulsion and the enhanced oral absorption. Int J Pharm, 246: 75-83,2002.

[5]. Kang BK, Lee JS, Chon SK, Jeong SY, Yuk SH, Khang G, Lee HB, and Cho SH. Development of self-microemulsifying drug delivery systems (SMEDDS) for oral bioavailability enhancement of simvastatin in beagle dogs. Int J Pharm, 274: 65-73,2004.

[6]. Ishiwa J, Sato T, Mimaki Y, Sashida Y, Yano M, and Ito A. A citrusflavonoid nobiletin suppresses production and gene expression of matrix metalloproteinase $9 /$ gelatinase $B$ in rabbit synovial fibroblasts. J Rheumatol, 27:20-25,2000. absorption in the gastrointestinal tract $[3,36]$. Moreover, the nobiletin SMEDDS and its dilutions were more stable than the nobiletin micelles(the data is not listed).

\section{CONCLUSIONS}

According to these results, several conclusions can be drawn. The nobiletin SMEDDS had a small particle size $(28.6 \pm 0.3 \mathrm{~nm})$ and a native charge. The $P_{\text {eff }}$ in jejunum at $15 \mu \mathrm{g} / \mathrm{mL}$ were significantly higher than that at $60 \mu \mathrm{g} / \mathrm{mL}(\mathrm{p}<0.01)$. Compared to the jejunum, duodenum and ileum, the higher $P_{\text {eff }}$ in colon were observed at the same concentration. The estimated absorption of nobiletin in human for the SMEDDS dilutions was higher than that for sub-microemulsions $(\mathrm{p}<0.01)$ and similar with that for the micelles $(p>0.05)$. Moreover, the nobiletin SMEDDS and its dilutions were more stable than the nobiletin micelles.

[7]. Lin N, Sato T, Takayama Y, Mimaki Y, Sashida Y, Yano M, and Ito A. Novel anti-inflammatory actions of nobiletin, a citrus polymethoxy flavonoid, on human synovial fibroblasts and mouse macrophages. Biochem Pharmacol, 65: 2065-2071, 2003.

[8]. Delaney B, Phillips K, Buswell D, Mowry B, Nickels D, Cox D, Wang HB, and Manthey J. Immunotoxicity of a standardized citrus polymethoxylated flavone extract. Food Chem Toxicol, 39: 1087-1094,2001.

[9]. Iwase Y, Takemura Y, Ju-ichi M, Yano M, Ito C, Furukawa H, Mukainaka T, Kuchidi M, Tokuda H, and Nishino H. Cancer chemopreventive activity of 3,5,6,7,8,3,4- heptametho-xyflavone from the peel of citrus plant. Cancer Lett,163: 7-9,2001.

[10]. Yoshimizu N, Otani Y, Saikawa Y, Kubota T, Yoshida M, Furukawa T, Kumai K, Kameyama K, Fujii M, Yano M, Sato T, Ito A, and Kitajima M. Anti-tumour effects of nobiletin, a citrus flavonoid, on gastric cancer include: antiproliferative effects, induction of apoptosis and cell cycle deregulation. Aliment Pharmacol Ther, 20: 95-101,2004.

[11]. Tanakaa S, Satoa T, Akimotoa N, Yanob M, and Ito A. Prevention of UVB-induced photoinflammation and photoaging by a polymethoxy flavonoid, nobiletin, in human keratinocytes in vivo and in vitro. Biochem Pharmacol, 68: 433-439,2004.

[12]. Thomas JC and Smriti SS. Intestinal permeability 
of chlorpyrifos using the single-pass intestinal perfusion method in the rat. Toxicology, 184: 125-133,2003.

[13]. Zakeri-Milani $\mathrm{P}$, Valizadeh $\mathrm{H}$, Tajerzadeh $\mathrm{H}$, Azarmi Y, Islambolchilar Ziba, Barzegar S, and Barzegar-Jalali M. Predicting human intestinal permeability using single-pass intestinal perfusion in rat. J Pham Pharmaceut Sci 10: 368-379,2007.

[14]. Laurent S, Kelly C, Lin P, Ken T, and Lori T. Evaluation of a single-pass intestinal- perfusion method in rat for the prediction of absorption in man. J Pharm Pharmacol 53: 1007-1013,2001.

[15]. You J, Li QP, Yu YW, and Cui FD. Absorption of zedoary oil in rat intestine using in situ single pass perfusion model. Acta Pharm Sin, 39: 849-853,2004.

[16]. Mario G and Giancarlo C. Theoretical considerations on the in vivo intestinal permeability determination by means of the single pass and recirculating techniques. Int $\mathrm{J}$ Pharm, 229: 95-105,2001.

[17]. Sutton SC, Rinaldi MT, and Vukovinsky KE. Comparison of the gravimetric, phenol red and 14C-PEG-3350 methods to determine water absorption in the rat single-pass intestinal perfusion model. AAPS Pharm Sci, 3: E25,2001.

[18]. Urban F, Monica J, and Hans L. Comparison between permeability coefficients in rat and human jejunum. Pharm Res,13: 1336-1342,1996.

[19]. Desesso JM and Jacobson CF. Anatomical and physiological parameters affecting gastrointestinal absorption in humans and rats. Food Chem Toxicol, 39: 209-228, 2001.

[20]. Amidon GL, kou J,. Elliott RL, and Lightfoot EN. Analysis of models for determining intestinal wall permeabilities. J Pharm Sci, 69: 1369-1373,1980.

[21]. Lindamark T, Nikkila, and Artursson P. Mechanisms of absorption enhancement by medium chain fatty acids in intestinal epithelial Caco-2 monolayers. J Pharmacol Exp, 275: 958-964,1995.

[22]. Charman WN, and Stella VJ. Transport of lipophilic molecules by the intestinal lymphatic system. Adv Drug Del Rev, 7: 1-14,1991.

[23]. Levitt MD, Aufderheide T, Fetzer CA, Bond JH, and Levitt DG. Use of carbon monoxide to measure luminal stirring in the rat gut. J Clin Invest, 74: 2056-2064, 1984.

[24]. Lennernas H. Does fluid flow across the intestinal mucosa affect quantitative oral drug absorption? Is it time for a reevaluation. Pharm Res, 12: 1573-1582,1995.

[25]. Fagerholm U, Nilsson D, Knutson L, and Lennernas H. Jejunal permeability in humans in vivo and rat in situ: investigation of molecular size selectivity and solvent drag. Acta Physiol
Scand, 165: 1-9,1999.

[26]. Ikegawa T, Ushigome F, Koyabu N, Morimoto S, Shoyama Y, Naito M, Tsuruo T, Ohtani H, and Sawada Y. Inhibition of P-glycoprotein by orange juice components, polymethoxy- flavones in adriamycin-resistant human myelogenous leukemia (K562/ADM) cells. Cancer Lett,160: 21-28, 2000

[27]. Fojo AT, Ueba K, Slamon DJ, Poplack DG, Gottesman MM, and Pastan I. Expression of multidrug-resistance gene in human tumors and tissues. Proc Natl Acd Sci USA, 84: 265-269,1987.

[28]. Trezise AE, Roman PR, Gill DR, Hyde SC, Sepulveda FV, Buchwald M, and Higgins CF. The multi-drug resistance and cystic fibrosis genes have complementary patterns of epithelial expression. EMBO J, 11: 4291-4303,1992.

[29]. Thomas YM, Daniel H, Richard AE, Hao T, Hien $\mathrm{N}$, and Pavel K. Mechanism of colonic permeation of inulin: is rat colon more permeable than small intestine. Gastroemterology, 108: 12-20,1995.

[30]. Chiou WL, and Barve A. Linear correlation of the fraction of the oral dose absorbed of 64 drugs between humans and rats. Pharm Res, 15: 1666-1671, 1998.

[31]. Majella EL, Karl AL, and Owen IC. Effect of intestinal fluid flux on ibuprofen absorption in the rat intestine. Int J Pharm, 309: 60-66,2006.

[32]. Lawrence XY, and Gordon LA. A compartmental absorption and transit model for estimating oral drug absorption. Int J Pharm, 186: 119-125,1999.

[33]. Mueller EA, Kovarik JM, Van Bree JB, Tetzloff W, Grevel J, and Kutz J. Improved dose linearity of cyclosporin pharmacokinetics from a microemulsion formulation. Pharm Res, 11: 301-304, 1994.

[34]. Jose GL, Jose Antonic PA, Rosa GA, Veronica B, Joel NR, Silvia ML, Jorge VO, Gilberto $\mathrm{CH}$, and Francisco JFM. Comparative bioavailability evaluation of two cyclosporine oral formulations in healthy Mexican volunteers. Arch Med Res, 30: 315-319,1999.

[35]. Humberstone AJ, and Charman WN. Lipid-based vehicles for the oral delivery of poorly water soluble drugs. Adv Drug Deliv Rev, 25: 103-128,1997.

[36]. Sha XY, Yan GJ, Wu YJ, Li JC, and Fang XL. Effect of self-microemulsifying drug delivery systems containing Labrasol on tight junctions in Caco-2 cells. Euro J Pharma Sci, 24: 477-486, 2005. 